\title{
Promoter hypomethylation of SKI in autoimmune pancreatitis
}

\author{
Yasuhiro Kinugawa $^{a}$, Takeshi Uehara ${ }^{a}$, , Kazuyuki Matsuda ${ }^{a}, Y$ Yukihiro Kobayashi ${ }^{a}$, \\ Tomoyuki Nakajima $^{a}$, Hideaki Hamano ${ }^{b}$, Shigeyuki Kawa ${ }^{\mathrm{d}}$, Kayoko Higuchi ${ }^{\mathrm{e}}$, Noriko Hosaka, \\ Satoshi Shiozawa ${ }^{g}$, Hiroki Ishigame ${ }^{g}$, Toshitsugu Nakamura ${ }^{\mathrm{h}}$, Yasuhiro Maruyama ${ }^{\mathrm{h}}$, \\ Koh Nakazawa ${ }^{\mathrm{i}}$, Masato Nakaguro ${ }^{\mathrm{j}}$, Kenji Sano ${ }^{\mathrm{k}}$, Hiroyoshi Ota ${ }^{\mathrm{a}, \mathrm{c}}$ \\ a Department of Laboratory Medicine, Shinshu University School of Medicine, Matsumoto, Japan \\ ${ }^{\mathrm{b}}$ Department of Gastroenterology, Shinshu University School of Medicine, Matsumoto, Japan \\ ${ }^{\mathrm{c}}$ Department of Biomedical Laboratory Medicine, Shinshu University School of Medicine, Matsumoto, Japan \\ d Internal Medicine, Matsumoto Dental University, Shiojiri, Japan \\ e Department of Pathology, Aizawa Hospital, Matsumoto, Japan \\ ${ }^{\mathrm{f}}$ Department of Pathology, Nagano Municipal Hospital, Nagano, Japan \\ ${ }^{g}$ Department of Pathology, Saku Central Hospital, Saku, Japan \\ h Department of Pathology, Suwa Red Cross Hospital, Suwa, Japan \\ ${ }^{i}$ Department of Clinical Laboratory, National Hospital Organization, Matsumoto Medical Center, Matsumoto Hospital, Matsumoto, Japan \\ ${ }^{\mathbf{j}}$ Department of Pathology and Laboratory Medicine, Nagoya University Hospital, Nagoya, Japan \\ ${ }^{\mathrm{k}}$ Department of Pathology, Iida Municipal Hospital, Iida, Japan
}

\section{A R T I C L E I N F O}

\section{Keywords:}

Autoimmune pancreatitis

Methylation abnormality

SKI

\begin{abstract}
A B S T R A C T
The relationship between methylation abnormality and autoimmune pancreatitis (AIP)—a representative IgG4related disease-has not yet been elucidated. We identified SKI might have a significant methylation abnormality in AIP through methylation array analysis using the Illumina Infinium Human Methylation 450K BeadChip array, and investigated the relationship of SKI with AIP clinicopathological features. The methylation rate of SKI was assessed by quantitative SYBR green methylation-specific PCR, and the degree of SKI expression in tissue specimens was assessed by immunohistochemistry in 10 AIP cases, 14 cases of obstructive pancreatitis area in pancreatic ductal adenocarcinoma (PDA) without a history of AIP, and 9 normal pancreas (NP) cases. The SKI methylation ratio was significantly lower in AIP than in PDA and NP. Additionally, the immunohistochemical staining-index (SI) score for SKI was significantly higher in AIP than NP, although there was no significant difference between AIP and PDA. There was a strong negative correlation between SI score and SKI methylation ratio, and between the serum concentrations of IgG4 and the SKI methylation ratio. There was a moderate positive correlation between the serum concentrations of IgG4 and SI. SKI is thought to be an oncogene indicating that SKI hypomethylation and carcinogenesis might be linked to AIP. Furthermore, the correlation between serum concentrations of IgG4 and SKI methylation levels suggest SKI might be involved in the pathogenesis of AIP. However, the role of SKI has not been clearly elucidated. Further studies are needed to understand further the function of SKI.
\end{abstract}

\section{Introduction}

IgG4-related disease (IgG4-RD) is a group of incompletely-understood inflammatory conditions that involve the formation of masses in multiple organs [1] and are characterized by high IgG4 serum values [1]. Autoimmune pancreatitis (AIP) is a representative IgG4-RD [2], and is pathologically characterized by lymphoplasmacytic inflammation and storiform fibrosis [3]. Pathologically, many IgG4-positive cells are observed in AIP lesions [4]. Steroid therapy is effective in IgG4-RDs, including AIP [5].

AIP is an autoimmune mediated chronic pancreatitis and its pathophysiology and clinical symptoms are characteristic of autoimmune diseases [6,7]. Autoimmune diseases such as rheumatoid arthritis and systemic lupus erythematosus (SLE) occur when tissue damage is caused by an autoimmune response. Recently, it was reported that most susceptibility gene polymorphisms of autoimmune diseases exist in enhancer regions [8]. This suggests the importance of the epigenome controlling the enhancer function [9]. Regarding DNA methylation,

\footnotetext{
* Corresponding author. Department of Laboratory Medicine, Shinshu University School of Medicine, 3-1-1 Asahi, Matsumoto 390-8621, Japan.

E-mail address: tuehara@shinshu-u.ac.jp (T. Uehara).
} 
analysis of epigenome-wide studies in patient specimens of SLE and rheumatoid arthritis (RA) reported the DNA hypomethylation of important genes involved in the onset of disease $[8,10]$. Because AIP is an autoimmune disease, methylation abnormalities may be involved in its pathogenesis.

Chronic inflammation causes epigenetic changes in genes. Methylation abnormalities sometimes induce carcinogenesis. Chronic pancreatitis is also an inflammatory condition, and 5\% of chronic pancreatitis patients develop carcinogenesis [11]. In chronic pancreatitis patients, the cancer risk is 13.3 times higher than in healthy subjects [11].

Methylation abnormalities in AIP may cause AIP or AIP itself may cause methylation abnormalities, and these methylation abnormalities might cause secondary changes such as carcinogenesis. However, methylation abnormalities of AIP have rarely been investigated. Genomic analysis by DNA methylation array is a commonly used method to evaluate methylation abnormalities associated with carcinogenesis and autoimmune diseases.

Using DNA methylation array results from AIP tissues, we identified $S K I$ as a candidate gene. We examined the methylation levels of SKI using Quantitative SYBR green methylation-specific PCR (QSG-MSP) and immunohistochemistry. This study evaluated the methylation levels of SKI with the clinicopathological data of AIP patients.

\section{Materials and methods}

\subsection{Patients and tissue samples}

We identified 10 AIP cases, 14 pancreatic ductal adenocarcinoma (PDA) cases without a history of AIP, and 9 normal pancreas (NP) cases that were resected for non-pancreatic carcinoma without a history of AIP, at Shinshu University Hospital or one of its affiliated hospitals from 1996 to 2013. In PDA cases, the obstructive pancreatitis (OP) area was used for various measurements. All AIP cases met the diagnostic criteria for AIP. Specimens were retrieved from archived tissue blocks. This study was approved by the Ethics Committee of Shinshu University, Japan. The demographic data of patients are provided in Table 1. All lesions were reviewed by two pathologists (T.U. and H.O.) for pathologic diagnosis and areas for manual macrodissection were selected by hematoxylin and eosin (H\&E)-stained tissue sections. For comparison with AIP and chronic inflammation other than AIP, the OP area was used for analyses. For comparison between AIP and normal pancreas without tumor, the following 9 normal samples were used: one case of injury due to accident and 8 cases of pancreatic tissue excised together with extra-pancreatic lesion. These cases did not have any neoplastic lesions in the pancreas.

\subsection{Methylation array}

Methylation analysis was performed on 10 AIP cases, 14 PDA cases, and $4 \mathrm{NP}$ cases using the Illumina Infinium Human Methylation 450K (HM450K) BeadChip array (Illumina, San Diego, CA). Images were

Table 1

Clinical features of patients with autoimmune pancreatitis (AIP), pancreatic ductal adenocarcinoma (PDA), and normal pancreas (NP).

\begin{tabular}{llll}
\hline & AIP & PDA & NP \\
\hline Age & $72.0(69.5-77.5)$ & $70.5(64.0-73.3)$ & $69.0(66.0-76.0)$ \\
Sex & & 11 & 6 \\
Male & 9 & 3 & 3 \\
Female & 1 & $23.0(20.0-33.0)$ & na \\
IgG4 (mg/dL) & $250.0(122.0-1415.0)$ & & \\
\hline
\end{tabular}

*Normal value for IgG4: < $70 \mathrm{mg} / \mathrm{dL}$ (cut-off: $135 \mathrm{mg} / \mathrm{dL}$ ).

AIP, autoimmune pancreatitis; PDA, pancreatic ductal adenocarcinoma; NP, normal pancreas; na, not available. obtained using the Genome Studio version 2011.1 methylation module (Illumina). The methylation score for each $\mathrm{CpG}$ group was represented as a beta-value according to the fluorescence intensity ratio. Beta-values may be any value between 0 (non-methylated) and 1 (completely methylated). The HM450K protocol required $1 \mu \mathrm{g}$ of bisulfite-converted DNA. For bisulfite treatment, genomic DNA was extracted from $5-\mu \mathrm{m}-$ thick, formalin-fixed and paraffin-embedded unstained tissue sections of 10 AIP, 14 PDA, and 4 NP samples, using the QIAamp DNA Minikit (Qiagen Inc., Valencia, CA). DNA was modified with sodium bisulfite treatment using the EZ DNA Methylation Kit (Zymo Research, Orange, CA) following the manufacturer's instructions.

\subsection{Quantitative SYBR green methylation-specific PCR (QSG-MSP)}

QSG-MSP was performed to quantify the levels of CpG DNA methylation of SKI using the Applied Biosystems 7500 real time PCR system (Applied Biosystems, Foster City, CA) as previously reported [12]. Primers for QSG-MSP were designed using Methyl Primer Express Software v1.0 (Applied Biosystems). The presence of CpG islands was determined using Methyl Primer Express v1.0 software (Applied Biosystems). Primer sequences were 5'-TAAAGTCGGGGATGGTAGGAC-3' (forward) and 5'-CGATCGCGATTCTTAAAAAAC-3' (reverse). Primers for the control ACTB (beta-actin) gene were $5^{\prime}$-TGGTGATGGAGGAGG TTTAGTAAGT-3' (forward) and 5'-AACCAATAAAACCTACTCCTCCCT TAA-3' (reverse) as previously described [12]. Quantitative PCR was performed in a $25-\mu \mathrm{L}$ reaction volume with $12.5 \mu \mathrm{L}$ of $2 \times$ SYBR Green PCR Master Mix (Applied Biosystems), 2.5 pmol of each primer, and $25 \mathrm{ng}$ of bisulfite-treated DNA sample. Thermal cycling was as follows: $95^{\circ} \mathrm{C}$ for $10 \mathrm{~min}$, and 40 cycles of $95^{\circ} \mathrm{C}$ for $15 \mathrm{~s}$, and $60^{\circ} \mathrm{C}$ for $1 \mathrm{~min}$. Only samples with amplification at the correct melting temperature were used for further analyses of methylation. The amount of methylated DNA (percentage of methylated reference) was calculated as follows: ratio of quantity of target gene to quantity of target gene of test sample divided by quantity of ACTB.

\subsection{Immunohistochemistry}

Immunohistochemical staining was performed on $4-\mu \mathrm{m}$-thick formalin-fixed, paraffin-embedded, tissue sections, using anti-SKI antibody (1:500; Santa Cruz, CA, USA). Briefly, deparaffinized tissue sections were treated with $0.3 \% \mathrm{H}_{2} \mathrm{O}_{2}$ for $30 \mathrm{~min}$ to inhibit endogenous peroxidase followed by antigen retrieval using microwave heating in EDTA/ Tris buffer ( $\mathrm{pH} \mathrm{9.0)} \mathrm{for} 25 \mathrm{~min}$. Overnight incubation at $4{ }^{\circ} \mathrm{C}$ with primary antibody against SKI diluted in bovine serum albumin was followed by incubation with the secondary antibody (NOVOLINK-Polymer Detection Systems) for $1 \mathrm{~h}$ at room temperature. Staining was developed by reaction with 3,3'-diaminobenzidine substrate-chromogen solution, followed by counterstaining with hematoxylin.

Staining results for the above antibody were evaluated by two pathologists (T.U. and H.O.) using a semi-quantitative method. We defined a staining-index (SI), which was calculated as the sum of two scores: an intensity score (IS) and a percentage score (PS). Previous studies showed positive expression of SKI by immunohistochemical analyses in both the cytoplasm and nucleus of malignant tumors, such as breast carcinoma and malignant melanoma [13-15]. The IS was scored on a four-tier scale based on the degree of cytoplasmic and nuclear staining intensity in most epithelial cells (score 0: no staining; 1: weak; 2: moderate; 3 : strong). The PS was scored on a four-tier scale based on the percentage of cells exhibiting positive cytoplasmic and nuclear staining among all target cells (score 0 : no staining; $1:<10 \%$; 2: $10-50 \% ; 3:>50 \%)$. Therefore, total SI scores ranged between 0 and 6.

\subsection{Statistical analysis}

Statistical analyses of clinical data were performed using the Chi- 
squared test or Wilcoxon rank sum test. Methylation array data, QSGMSP data, and immunohistochemistry data were analyzed using the Wilcoxon rank sum test. The Spearman's rank correlation coefficient was used to assess correlations. Statistical analyses were conducted using JMP software version 10 (SAS Institute Japan, Japan). $P$ values of $<0.05$ were considered significant.

\section{Results}

\subsection{Clinical features}

There were no significant differences in gender between AIP and PDA cases $(P=0.4589)$, or AIP and NP cases $(P=0.2129)$ (Table 1$)$. There were no significant differences in age between AIP (mean 72 years, range 69.5-77.5) and PDA (mean 70.5 years, range 64.0-73.3) cases $(P=0.2069)$, or AIP (mean 72 years, range 69.5-77.5) and NP (mean 69 years, range 66.0-76.0) cases $(P=0.4128)$ (Table 1$)$.

The serum concentrations of IgG4 were measured in nine AIP (mean $250 \mathrm{mg} / \mathrm{dL}$, range 122-1415) and $11 \mathrm{PDA}$ (mean $23 \mathrm{mg} / \mathrm{dL}$, range 20-33) cases (Table 1). Seven AIP cases had high serum IgG4 levels (cut-off, $135 \mathrm{mg} / \mathrm{dL}$ ). All PDA cases had low serum levels. The serum concentrations of IgG4 were not measured in NP cases.

\subsection{Histological findings}

All AIP cases had lymphoplasmacytic inflammation, storiform fibrosis, and abundant IgG4-positive cells ( $\geq 10$ cells/high-power field) (Fig. 1A and B). No PDA or NP cases exhibited the features of IgG4-RD.

\subsection{Beta-values of methylation array and the methylation rate of QSG-MSP}

We conducted a methylation array to identify hypomethylated genes in AIP. SKI returned high beta-values in NP, representing hypermethylation, and low beta-values in AIP, representing hypomethylation, using HM450K. Notably, SKI had the largest difference between beta-values in AIP and NP. Therefore, we compared the methylation
A, The $S K I$ beta-value of methylation array

B, The $S K I$ methylation rate of QSG-MSP
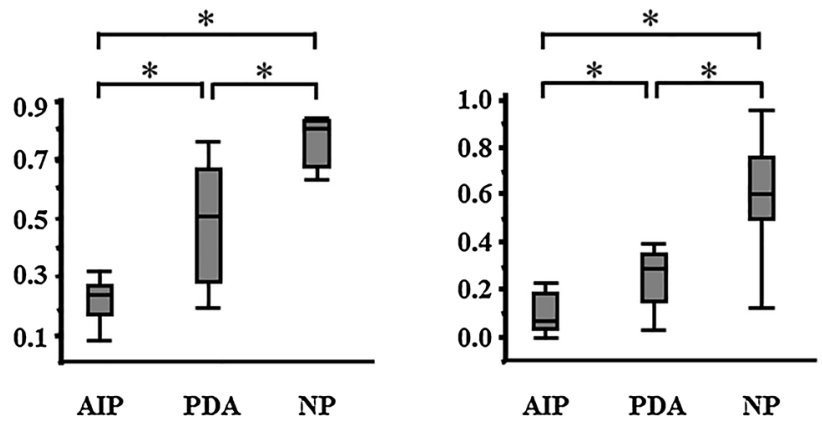

C, SI score

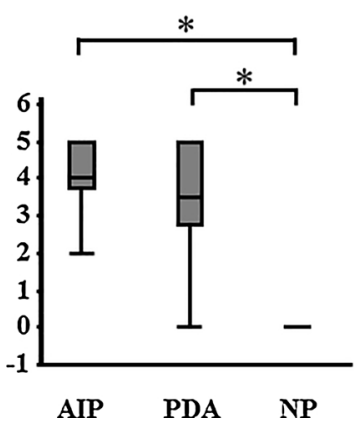

Fig. 2. Box plot of the SKI beta-values from methylation array, SKI methylation rates of QSG-MSP, and SI scores in patients with autoimmune pancreatitis (AIP) vs. obstructive pancreatitis area in pancreatic ductal adenocarcinoma (PDA) and normal pancreas (NP).

levels of SKI with clinicopathological data from AIP cases.

The SKI beta-value was significantly lower in AIP (mean 0.24, range 0.17-0.27) compared with PDA (mean 0.50, range 0.28-0.67) and NP (mean 0.81, range 0.66-0.84) cases $(P=0.0014$ and $P=0.0058$ ) (Fig. 2A). The SKI beta-value for PDA cases (mean 0.50, range,
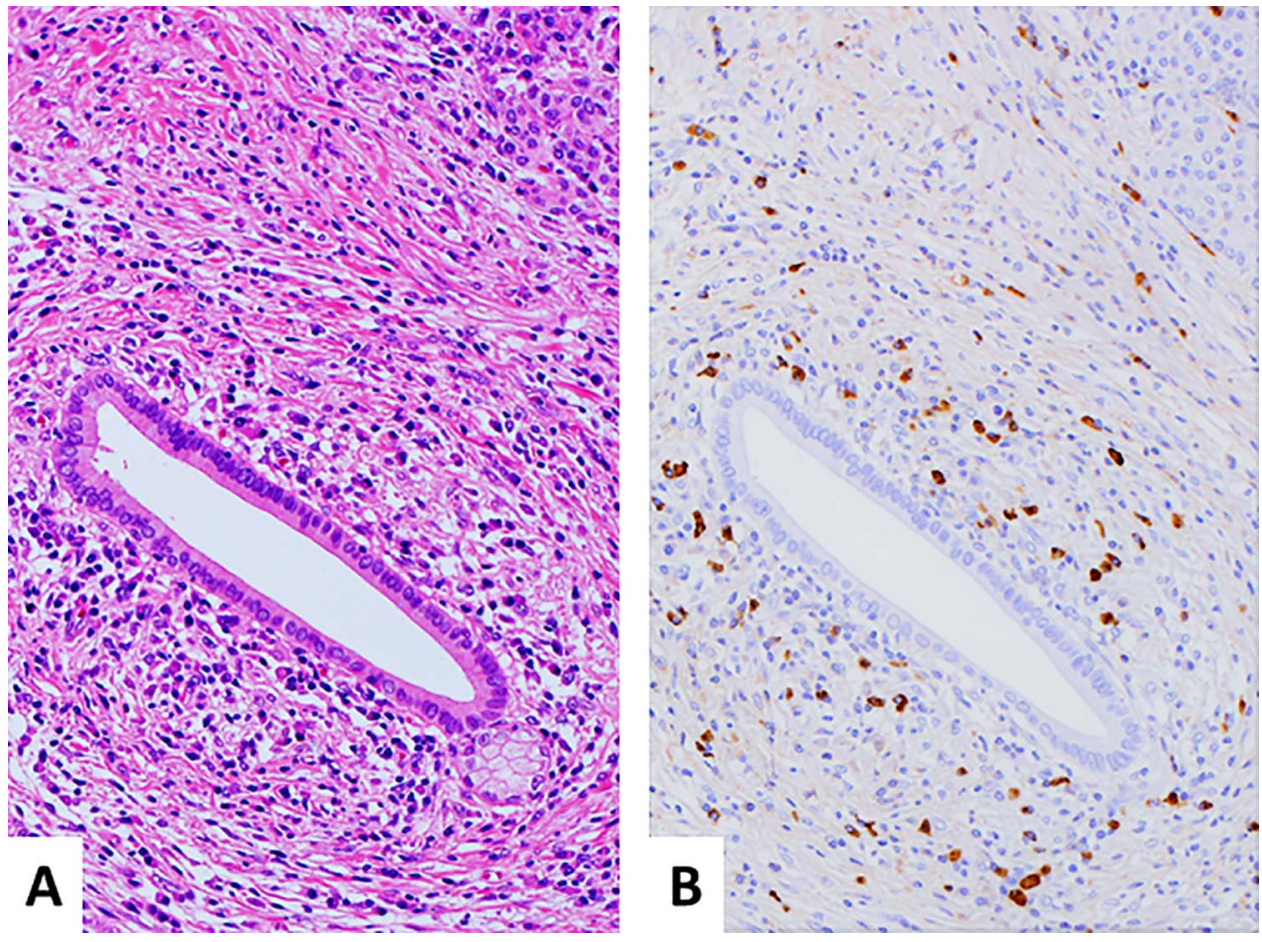

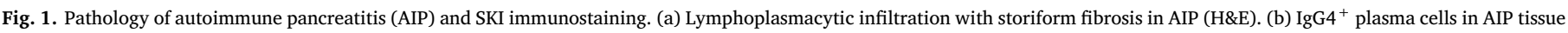
(IgG4 immunostaining). Original magnification: $\times 25$. 

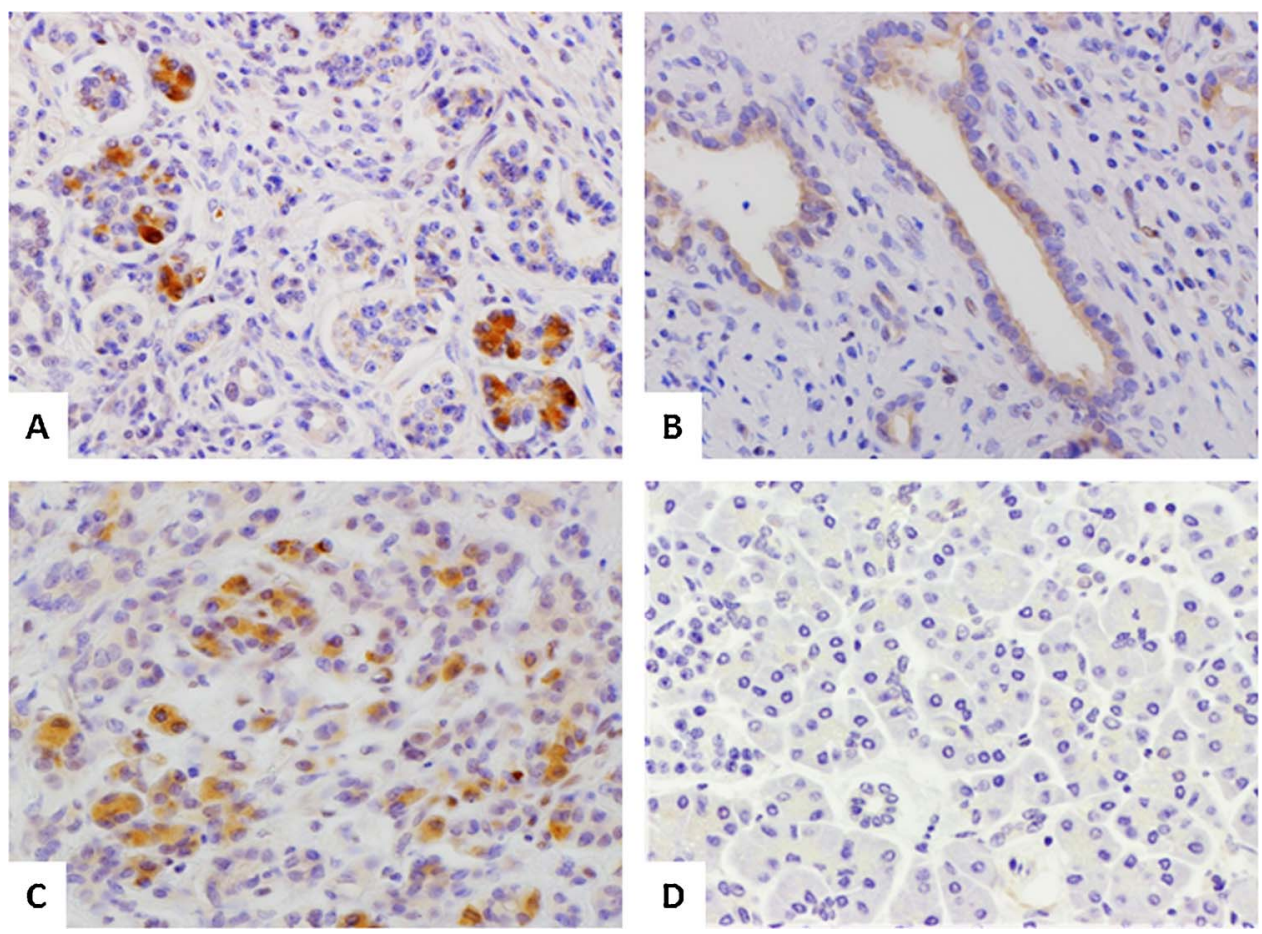

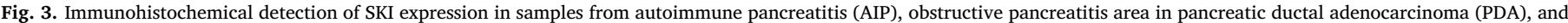
normal pancreas (NP). SKI staining in acinar cells (A) and pancreatic duct cells (B) in AIP. SKI staining in acinar cells (C) in PDA and NP (D). Original magnification: $\times 25$.

0.28-0.67) was also significantly lower than that of NP (mean 0.81 , range, 0.66-0.84) cases ( $P=0.0169)$ (Fig. 2A).

By QSG-MSP, the SKI methylation ratio was significantly lower in AIP cases (mean 7.11, range 3.35-18.45) compared with PDA (mean 28.89, range 14.87-35.15) and NP (mean 60.55, range 49.72-76.11) cases $(P=0.0041$ and $P=0.0011)$ (Fig. 2B). The $S K I$ methylation ratio in PDA (mean 28.89, range 14.87-35.15) cases was also significantly lower than that of NP (mean 60.55, range 49.72-76.11) cases $(P=0.0015)$ (Fig. 2B).

\subsection{Immunohistochemistry of SKI}

Positive SKI signals were identified in the cytoplasm of acinar and pancreatic duct cells in both AIP (Fig. 3A and B) and PDA cases (Fig. 3C). The pattern of SKI-positive cells varied from diffusely positive to scattered positive. The density of positive cells also varied from strong to weak. No positive cells were identified in NP samples (Fig. 3D).

The SI score of immunohistochemical staining was significantly higher in AIP (mean 4.00, range 3.75-5.00) samples than in NP (no signal) samples $(P=0.0001)$ (Fig. $2 C)$. There was no significant difference in SI score between AIP (mean 4.00, range 3.75-5.00) samples and PDA (mean 3.50, range 2.75-5.00) samples $(P=0.2610$ ) (Fig. 2C). The SI score was significantly higher in PDA (mean 3.50, range, 2.75-5.00) samples than in NP (no staining) $(P=0.0001)$ (Fig. $2 \mathrm{C}$ ).

\subsection{Correlations between clinicopathological data, QSG-MSP methylation} rate, and SI score in AIP

There was no statistically significant correlation between SI score and the SKI methylation ratio in AIP cases $(\mathrm{r}=-0.6142, P=0.0785)$ (Table 2), although they had a tendency towards a moderate negative correlation. There was strong negative correlation between the serum concentrations of IgG4 and the SKI methylation ratio $(\mathrm{r}=-0.8503$, $P=0.0075$ ) (Table 2). There was a moderate positive correlation between the serum concentrations of IgG4 and SI $(r=0.6739$, $P=0.0466$ ) (Table 2). There was no correlation between age and the
Table 2

Correlations between clinicopathological data, beta-values of the methylation array, methylation rates of QSG-MSP, and the SI scores in AIP.

\begin{tabular}{|c|c|c|c|c|c|}
\hline & & Age & IgG4 & Methylation ratio & SI score \\
\hline \multirow[t]{2}{*}{ Age } & $\mathrm{r}$ & & & & \\
\hline & $P$ & & & & \\
\hline \multirow[t]{2}{*}{ IgG4 } & $\mathrm{r}$ & 0.0588 & & & \\
\hline & $P$ & 0.8805 & & & \\
\hline \multirow{2}{*}{$\begin{array}{l}\text { Methylation } \\
\text { ratio }\end{array}$} & $\mathrm{r}$ & -0.443 & -0.8503 & & \\
\hline & $P$ & 0.2323 & $0.0075^{*}$ & & \\
\hline \multirow[t]{2}{*}{ SI score } & $\mathrm{r}$ & 0.0358 & 0.6739 & -0.6142 & \\
\hline & $P$ & 0.9218 & $0.0466^{*}$ & 0.0785 & \\
\hline
\end{tabular}

r, Spearman's rank correlation coefficient; $P, p$-value. Methylation ratio, the methylation rate of QSG-MSP. * $P<0.05$.

SKI methylation ratio $(\mathrm{r}=-0.4430, P=0.2323)$, or age and SI ( $\mathrm{r}=0.0358, P=0.9218$ ) (Table 2). There was no correlation between serum IgG4 concentrations and age $(\mathrm{r}=0.0588, P=0.8805)$ (Table 2$)$.

\section{Discussion}

We identified a novel methylation abnormality in SKI characterized by hypomethylation in AIP patients. There have been few reports regarding methylation array analysis in AIP, and there has been no previous report on the role of SKI in AIP.

Differences in the analysis methods used might explain the failure to observe a correlation between the SKI beta-values from the methylation array and the QSG-MSP-based SKI methylation rate values in AIP. A correlating trend between SI score and the SKI methylation rate suggests that immunostaining is an important tool for assessing SKI methylation abnormalities, and the evaluation of methylation abnormalities by immunostaining was previously reported for many genes [16].

Our study identifies a clear difference in the methylation of SKI between AIP and NP cases and between PDA and NP cases. Inflammatory changes in AIP and PDA may affect the methylation levels of SKI. However, as there may be a difference in the methylation 
levels of SKI between AIP and PDA, additional factors may also be involved. Age might be an important factor that influences methylation abnormalities, but there were no differences in patient age between groups in our study.

SKI encodes a transforming protein of the avian Sloan-Kettering retrovirus that was originally shown to exhibit oncogenic activity $[17,18]$. In the early stages of tumor development, SKI shows oncogenic activity in repressing the tumor suppressive activities of TGF-beta/ Smads in inducing apoptosis and cell growth arrest [18]. In contrast, other findings showed that SKI contains anti-oncogenic activity in mammalian cells in the later stages of malignancy involved in invasion and metastasis $[18,19]$. SKI is overexpressed in pancreatic cancer [20], melanoma [15,21], esophageal cancer [22], colorectal cancer [23], and acute myeloid leukemia [24]. In AIP, SKI may function as an oncogene, which reflects its activity at early stages of tumor development. Although SKI might be an oncogene, its carcinogenic potential might be suppressed by the hypermethylation of its promoter region. Hypomethylation of the SKI promoter may result from the characteristic chronic inflammation present in AIP [25] and the hypomethylation of oncogenes was reported to be associated with carcinogenesis [26]. Therefore, SKI hypomethylation may be involved in carcinogenesis in AIP.

Only one previous report has described a relationship between SKI and pancreatic cancer, but no study has provided a detailed description on the expression pattern of SKI by immunohistochemistry; however, normal pancreas seems to show negative SKI expression [20]. In contrast, other reports in breast carcinoma and malignant melanoma cases showed positive SKI immunohistochemical staining in the cytoplasm as well as nucleus of tumor cells [13-15]. In our study, SKI was expressed in acinar cells as determined by immunostaining in OP and AIP, indicating that SKI may be associated with pancreatic acinar cell carcinoma. Similarly, SKI expression is also detectable in pancreatic ductal cells, and may therefore be involved in pancreatic duct adenocarcinoma. There are several case reports regarding carcinogenesis in AIP [27]. While reports that KRAS abnormalities are present in carcinogenesis with AIP exist [28,29], it remains controversial whether KRAS is functionally involved in carcinogenesis associated with AIP. To date, only a single report by Kinugawa et al. [12] has proposed a relationship between AIP-associated carcinogenesis and methylation abnormalities.

The results of the current study suggest there may be an association between the serum concentrations of IgG4 and SKI methylation levels. Abnormal methylation of SKI may be involved in the onset of AIP, because $S K I$ also influences inflammatory and immune responses via TGF-beta 1 [30]. Systemic lupus erythematosus, multiple sclerosis, autoimmune thyroiditis and other autoimmune diseases have been associated with the hypomethylation of genes [31-33]. In autoimmune thyroiditis, hypomethylation of the ICAM1 promoter region increases the expression of ICAM1 and may be involved in the onset of autoimmune thyroiditis [33]. Just as DNA methylation is a source of genetic variation that affects the risk of autoimmune diseases in other autoimmune diseases, SKI methylation abnormality in AIP may also be an important factor in AIP risk.

A limitation of our study is that it remains unknown whether SKI functions to drive mutations in the carcinogenesis process. To determine the involvement of SKI in carcinogenesis, SKI-expressing gene transfer in genetically modified mice is required. Furthermore, the accumulation of a larger number of samples from AIP cases is warranted for data that are more robust.

In conclusion, our study suggests that SKI hypomethylation might play an important role in AIP, and related carcinogenesis. However, the role of SKI has not been clearly elucidated and further studies are needed to understand further the function of SKI.

\section{Disclosure/conflict of interest}

The authors declare no conflict of interest.

\section{Acknowledgements}

We are grateful to Masanobu Momose, Yasuyo Shimojo, Naoko Ogiwara, Mieko Horikawa, Akiko Inamura, Chitoshi Arai, Rie Nakata, Souhei Koiwai, and Marina Nuno at Shinshu University Hospital for their excellent technical assistance. This study was supported by JSPS KAKENHI Grant Number JP17K08738. We thank Edanz Group (www. edanzediting.com/ac) for editing a draft of this manuscript.

\section{References}

[1] J.H. Stone, Y. Zen, V. Deshpande, IgG4-related disease, N. Engl. J. Med. 366 (2012) 539-551.

[2] H. Hamano, S. Kawa, A. Horiuchi, H. Unno, N. Furuya, T. Akamatsu, M. Fukushima, T. Nikaido, K. Nakayama, N. Usuda, K. Kiyosawa, High serum IgG4 concentrations in patients with sclerosing pancreatitis, N. Engl. J. Med. 344 (2001) 732-738.

[3] K. Kawaguchi, M. Koike, K. Tsuruta, A. Okamoto, I. Tabata, N. Fujita, Lymphoplasmacytic sclerosing pancreatitis with cholangitis: a variant of primary sclerosing cholangitis extensively involving pancreas, Hum. Pathol. 22 (1991) 387-395.

[4] T. Kamisawa, N. Funata, Y. Hayashi, Y. Eishi, M. Koike, K. Tsuruta, A. Okamoto, N. Egawa, H. Nakajima, A new clinicopathological entity of IgG4-related autoimmune disease, J. Gastroenterol. 38 (2003) 982-984.

[5] H. Umehara, K. Okazaki, Y. Masaki, M. Kawano, M. Yamamoto, T. Saeki, S. Matsui, T. Sumida, T. Mimori, Y. Tanaka, K. Tsubota, T. Yoshino, S. Kawa, R. Suzuki, T. Takegami, N. Tomosugi, N. Kurose, Y. Ishigaki, A. Azumi, M. Kojima, S. Nakamura, D. Inoue, A novel clinical entity, IgG4-related disease (IgG4RD): general concept and details, Mod. Rheumatol. 22 (2012) 1-14.

[6] S. Detlefsen, J.H. Brasen, G. Zamboni, P. Capelli, G. Kloppel, Deposition of complement C3c, immunoglobulin (Ig)G4 and IgG at the basement membrane of pancreatic ducts and acini in autoimmune pancreatitis, Histopathology 57 (2010) $825-835$.

[7] O. Cai, S. Tan, From Pathogenesis, Clinical manifestation, and diagnosis to treat ment: an overview on autoimmune pancreatitis, Gastroenterol. Res. Pract. 2017 (2017) 3246459.

[8] K.K. Farh, A. Marson, J. Zhu, M. Kleinewietfeld, W.J. Housley, S. Beik, N. Shoresh, H. Whitton, R.J. Ryan, A.A. Shishkin, M. Hatan, M.J. Carrasco-Alfonso, D. Mayer, C.J. Luckey, N.A. Patsopoulos, P.L. De Jager, V.K. Kuchroo, C.B. Epstein, M.J. Daly, D.A. Hafler, B.E. Bernstein, Genetic and epigenetic fine mapping of causal autoimmune disease variants, Nature 518 (2015) 337-343.

[9] D. Hammaker, J.W. Whitaker, K. Maeshima, D.L. Boyle, A.H. Ekwall, W. Wang, G.S. Firestein, LBH gene transcription regulation by the interplay of an enhancer risk allele and DNA methylation in rheumatoid arthritis, Arthr. Rheumatol. 68 (2016) 2637-2645.

[10] C.M. Hedrich, T. Rauen, S.A. Apostolidis, A.P. Grammatikos, N. Rodriguez Rodriguez, C. Ioannidis, V.C. Kyttaris, J.C. Crispin, G.C. Tsokos, Stat3 promotes IL10 expression in lupus $\mathrm{T}$ cells through trans-activation and chromatin remodeling, Proc. Natl. Acad. Sci. U. S. A. 111 (2014) 13457-13462.

[11] S. Raimondi, A.B. Lowenfels, A.M. Morselli-Labate, P. Maisonneuve, R. Pezzilli, Pancreatic cancer in chronic pancreatitis; aetiology, incidence, and early detection, Best Pract. Res. Clin. Gastroenterol. 24 (2010) 349-358.

[12] Y. Kinugawa, T. Uehara, K. Sano, K. Matsuda, Y. Maruyama, Y. Kobayashi, T. Nakajima, H. Hamano, S. Kawa, K. Higuchi, N. Hosaka, S. Shiozawa, H. Ishigame, H. Ota, Methylation of Tumor Suppressor Genes in Autoimmune Pancreatitis, Pancreas, 2017.

[13] I. Theohari, I. Giannopoulou, C. Magkou, A. Nomikos, S. Melissaris, L. Nakopoulou, Differential effect of the expression of TGF-beta pathway inhibitors, Smad-7 and Ski, on invasive breast carcinomas: relation to biologic behavior, APMIS 120 (2012) 92-100.

[14] B. Boone, M. Haspeslagh, L. Brochez, Clinical significance of the expression of c-ski and SnoN, possible mediators in TGF-beta resistance, in primary cutaneous melanoma, J. Dermatol. Sci. 53 (2009) 26-33.

[15] J.A. Reed, E. Bales, W. Xu, N.A. Okan, D. Bandyopadhyay, E.E. Medrano, Cytoplasmic localization of the oncogenic protein Ski in human cutaneous melanomas in vivo: functional implications for transforming growth factor beta signaling, Cancer Res. 61 (2001) 8074-8078.

[16] H. Ushiku, K. Yamashita, H. Kawamata, M. Waraya, H. Katoh, K. Yokoi, T. Tanaka, S. Ishii, N. Nishizawa, M. Kikuchi, N. Minatani, K. Kojo, H. Tajima, R. Nishiyama, T. Kaizu, Y. Kumamoto, M. Watanabe, Homeobox-only protein expression is a critical prognostic indicator of pancreatic neuroendocrine tumor and is regulated by promoter DNA hypermethylation, Pancreas 45 (2016) 1255-1262.

[17] Y. Li, C.M. Turck, J.K. Teumer, E. Stavnezer, Unique sequence, ski, in SloanKettering avian retroviruses with properties of a new cell-derived oncogene, J. Virol. 57 (1986) 1065-1072.

[18] J. He, S.B. Tegen, A.R. Krawitz, G.S. Martin, K. Luo, The transforming activity of Ski and SnoN is dependent on their ability to repress the activity of Smad proteins, J. Biol. Chem. 278 (2003) 30540-30547.

[19] J. Deheuninck, K. Luo, Ski and SnoN, potent negative regulators of TGF-beta signaling, Cell Res. 19 (2009) 47-57.

[20] T.R. Heider, S. Lyman, R. Schoonhoven, K.E. Behrns, Ski promotes tumor growth through abrogation of transforming growth factor-beta signaling in pancreatic cancer, Ann. Surg. 246 (2007) 61-68. 
[21] J.A. Reed, Q. Lin, D. Chen, I.S. Mian, E.E. Medrano, SKI pathways inducing progression of human melanoma, Cancer Metastasis Rev, 24 (2005) 265-272.

[22] M. Fukuchi, M. Nakajima, Y. Fukai, T. Miyazaki, N. Masuda, M. Sohda, R. Manda, K. Tsukada, H. Kato, H. Kuwano, Increased expression of c-Ski as a co-repressor in transforming growth factor-beta signaling correlates with progression of esophageal squamous cell carcinoma, Int. J. Cancer 108 (2004) 818-824.

[23] M. Buess, L. Terracciano, J. Reuter, P. Ballabeni, J.L. Boulay, U. Laffer, U. Metzger, R. Herrmann, C. Rochlitz, Amplification of SKI is a prognostic marker in early colorectal cancer, Neoplasia 6 (2004) 207-212.

[24] M. Ritter, D. Kattmann, S. Teichler, O. Hartmann, M.K. Samuelsson, A. Burchert, J.P. Bach, T.D. Kim, B. Berwanger, C. Thiede, R. Jager, G. Ehninger, H. Schafer, N. Ueki, M.J. Hayman, M. Eilers, A. Neubauer, Inhibition of retinoic acid receptor signaling by Ski in acute myeloid leukemia, Leukemia 20 (2006) 437-443.

[25] B. Cho, H. Lee, S. Jeong, Y.J. Bang, H.J. Lee, K.S. Hwang, H.Y. Kim, Y.S. Lee, G.H. Kang, D.I. Jeoung, Promoter hypomethylation of a novel cancer/testis antigen gene CAGE is correlated with its aberrant expression and is seen in premalignant stage of gastric carcinoma, Biochem. Biophys. Res. Commun. 307 (2003) 52-63.

[26] K. Kawaguchi, A. Kinameri, S. Suzuki, S. Senga, Y. Ke, H. Fujii, The cancer-promoting gene fatty acid-binding protein 5 (FABP5) is epigenetically regulated during human prostate carcinogenesis, Biochem. J 473 (2015) 449-461.

[27] H. Inoue, H. Miyatani, Y. Sawada, Y. Yoshida, A case of pancreas cancer with autoimmune pancreatitis, Pancreas 33 (2006) 208-209.

[28] T. Kamisawa, K. Tsuruta, A. Okamoto, S. Horiguchi, Y. Hayashi, X. Yun, T. Yamaguchi, T. Sasaki, Frequent and significant K-ras mutation in the pancreas, the bile duct, and the gallbladder in autoimmune pancreatitis, Pancreas 38 (2009) 890-895.

[29] A. Khalid, J. Dewitt, N.P. Ohori, J.H. Chen, K.E. Fasanella, M. Sanders, K.M. McGrath, M. Nikiforova, EUS-FNA mutational analysis in differentiating autoimmune pancreatitis and pancreatic cancer, Pancreatology 11 (2011) 482-486.

[30] B.L. Zampieri, J.M. Biselli-Perico, J.E. de Souza, M.C. Burger, W.A. Silva Junior, E.M. Goloni-Bertollo, E.C. Pavarino, Altered expression of immune-related genes in children with down syndrome, PLoS One 9 (2014) e107218.

[31] M.A. Jeffries, A.H. Sawalha, Autoimmune disease in the epigenetic era: how has epigenetics changed our understanding of disease and how can we expect the field to evolve? Expert Rev. Clin. Immunol. 11 (2015) 45-58.

[32] J. Field, A. Fox, M.A. Jordan, A.G. Baxter, T. Spelman, M. Gresle, H. Butzkueven, T.J. Kilpatrick, J.P. Rubio, Interleukin-2 receptor-alpha proximal promoter hypomethylation is associated with multiple sclerosis, Genes Immun. 18 (2017) 59-66.

[33] T. Liu, J. Sun, Z. Wang, W. Yang, H. Zhang, C. Fan, Z. Shan, W. Teng, Changes in the DNA methylation and hydroxymethylation status of the intercellular adhesion molecule 1 gene promoter in thyrocytes from autoimmune thyroiditis patients, Thyroid 27 (2017) 838-845. 